\title{
Evaluation of Fatigue Induced Damages in CFRP Laminates With Thermography Technique
}

\author{
K. Panbarasu*, S. Kalyana Sundaram*, V.R. Ranganath*, Raghu V. Prakash** \\ * CSIR-National Aerospace Laboratories, Bengaluru - 560017, India. \\ ** Dept. of Mechanical Engg. Indian Institute of Technology, Chennai, India \\ Email : kalyan@nal.res.in
}

\begin{abstract}
Prolific usage of continuous fiber composite materials and their variants in aerospace industry has attracted the attention of materials researchers towards understanding the fatigue behavior of this material. Wide spread literature on this subject provides the information that initial stages of fatigue, introduces complex and clusters of micro damages such as fibre - matrix interface failure, matrix failure, uneven fibre breaks etc. In composite laminates detection and evaluation of these early stage micro-damages due to fatigue on composite materials poses challenges due to the limitation of sensitivity levels of NDE technique such as ultrasonic methods that are currently employed for the composite laminates.

Paper presented here provides results of a study on detection of early stage fatigue damages in CFRP laminates with thermography technique. CFRP specimens were subjected to fatigue loading with a maximum stress level to the failure stress. Tension - Tension cycles were applied on a set of CFRP specimens. The specimens were subjected to IR thermography intermediately during fatigue cycles to understand the effect of progressive damage on the patterns of thermograms generated. Transient thermography was used in both transmission and reflection mode in this evaluation. Transmitted and reflected heat flux from the specimens have been measured through high end mid wave infrared camera and analyzed. Trend analysis of thermograms of fatigued specimens shows variation in time dependent heat flux from the specimens in, both, transmitted and reflected flux. Attempts were made to quantify the early stage fatigue damages in this study are also presented.
\end{abstract}

Keywords: Fatigue damage, composites, CFRP, matrix failure, thermography 